\title{
RELATO DE EXPERIÊNCIA NA CAPACITAÇÃO EM ATENDIMENTO ODONTOLÓGICO DE URGÊNCIA E EMERGÊNCIA
}

\author{
Gisele Jung Franciscatto \\ Universidade Federal de Santa Maria \\ giselejfranciscatto@hotmail.com \\ Renata Dornelles Morgental \\ Universidade Federal de Santa Maria \\ remorgental@hotmail.com \\ Rafaela Oliveira Pilecco \\ Universidade Federal de Santa Maria \\ rafaela-pilecco@hotmail.com
}

\begin{abstract}
Resumo
Esse artigo apresenta o relato de experiência a respeito do projeto de Capacitação em Atendimento Odontológico de Urgência e Emergência na Universidade Federal de Santa Maria. O tratamento de urgência e emergência pode ser descrito como a abordagem inicial de um paciente que se encontra em situação de dor ou anormalidade, abrangendo afecções pulpares, periapicais, periodontais ou traumáticas, necessitando intervenções clínicas e/ou cirúrgicas. Esses atendimentos fazem parte da rotina do cirurgião-dentista, logo, o projeto surgiu com o objetivo de capacitar alunos do Curso de Odontologia que têm pouca experiência e contato durante a graduação com tais procedimentos. $\mathrm{O}$ projeto também tem objetivo de ofertar à população, que busca atendimento nas clínicas da Universidade, uma opção de um serviço destinado exclusivamente para esses atendimentos, visto que são poucas vagas ofertadas pelas clínicas usuais da graduação. Com início em março de 2017, funcionando em dois turnos semanais, conta com alunos de graduação, professores, uma odontóloga e alunos de pós-graduação. O atendimento de urgência busca, principalmente, o alívio da dor, eliminando imediatamente sua causa, e restauração da função dentária e encaminhamento do paciente para realização do tratamento odontológico definitivo adequado. Até dezembro de 2017, com cerca de 10 meses de projeto, aproximadamente 80 pacientes receberam atendimento, obtendo suas queixas sanadas e sendo acolhidos pela Universidade. Cerca de $77 \%$ dos atendimentos envolveram alguma intervenção endodôntica.
\end{abstract}

Palavras-chave: Tratamento de urgência odontológica. Dor dental. Traumatismo dentário.

\section{EXPERIENCE REPORT IN TRAINING IN URGENCY AND EMERGENCY TREATMENT}

\begin{abstract}
This article presents the experience report about the project of Training in Dental Emergency Care at the Federal University of Santa Maria. Urgent and emergency treatment can be described as the initial approach of a patient who is in pain or abnormality, including pulp, periapical, periodontal or traumatic affections, requiring clinical and/or surgical interventions. These appointments are part of the dentist's routine, so the project came up with the objective of training dentistry students who have little experience and contact during graduation with such procedures. The project also aims to offer the population, who seeks care in the clinics of the University, an option of a service exclusively for these kind of situation, since there are few possibilities offered by the usual clinics of the graduation. Beginning in March 2017, working in two weekly shifts, it counts with undergraduate students, teachers, a dentist and graduate students. Urgent care mainly seeks to relieve pain, eliminating its cause immediately, and restoring the dental function and referral of the patient to perform the
\end{abstract}


definitive dental treatment. By December 2017, with approximately 10 months of project, approximately 80 patients received care, obtaining their complaints healed and being welcomed by the University. About $77 \%$ of the visits involved some endodontic intervention.

Keywords: Urgency dental treatment. Dental pain. Dental trauma.

\section{RELATO DE EXPERIENCIA EN LA CAPACITACIÓN EN ATENCIÓN ODONTOLÓGICA DE URGENCIA Y EMERGENCIA}

\section{Resumen}

Este artículo presenta el relato de experiencia acerca del proyecto de Capacitación en Atención Odontológica de Urgencia y Emergencia en la Universidad Federal de Santa María. El tratamiento de urgencia y emergencia puede ser descrito como el abordaje inicial de un paciente que se encuentra en situación de dolor o anormalidad, que abarca afecciones pulparas, periapicales, periodontales o traumáticas, necesitando intervenciones clínicas y / o quirúrgicas. En la mayoría de los casos, la mayoría de las personas que sufren de depresión, que no se sienten satisfechas. El proyecto también tiene el objetivo de ofrecer a la población, que busca atención en las clínicas de la Universidad, una opción de un servicio destinado exclusivamente a esas atenciones, ya que son pocas vacantes ofrecidas por las clínicas usuales de la graduación. A comienzos en marzo de 2017, funcionando en dos turnos semanales, cuenta con alumnos de graduación, profesores, una odontóloga y alumnos de postgrado. La atención de urgencia busca, principalmente, el alivio del dolor, eliminando inmediatamente su causa, y restauración de la función dental y encaminamiento del paciente para realización del tratamiento odontológico definitivo adecuado. Hasta diciembre de 2017, con cerca de 10 meses de proyecto, aproximadamente 80 pacientes recibieron atención, obteniendo sus quejas sanadas y siendo acogidos por la Universidad. Cerca del $77 \%$ de las atenciones involucraron alguna intervención endodóntica.

Palabras claves: Tratamiento de urgencia odontológica. Dolor dental. Traumatismo dental. 


\section{INTRODUÇÃO}

Atendimentos de urgências e emergências em Odontologia são práticas frequentes no cotidiano de serviços públicos ou privados. Embora a Odontologia atual busque cada vez mais enfatizar a prevenção de patologias dentárias e a promoção de saúde, o serviço odontológico oferecido em serviços públicos no Brasil não é suficiente para atender grande parte da população, fazendo com que esses indivíduos fiquem desassistidos em atividades de prevenção, detecção e tratamento precoce de cárie dentária e outras doenças (ALBUQUERQUE, 2016). Diante disso, enfrenta-se uma realidade na qual, frente a falta de informação e recursos, as pessoas buscam tratamento odontológico na grande maioria das vezes devido a um sofrimento ou alteração funcional (SAKAI et al., 2005).

O tratamento de urgência e emergência odontológica pode ser descrito como a abordagem inicial de um paciente que se encontra em situação de dor ou anormalidade, abrangendo afecções pulpares, periapicais, periodontais ou traumáticas, necessitando intervenções clínicas e/ou cirúrgicas. Segundo a Associação Internacional para Estudos da Dor (IASP), dor conceitua-se como uma experiência sensorial e emocional desagradável associada a dano tecidual real ou potencial, ou descrita em termos de tal dano. É listado como uma das principais causas de impacto psicossocial, físico e econômico, influenciando atividades diárias e a qualidade de vida dos indivíduos. (CAVALHEIRO et al., 2015; CONSTANTE et al., 2012; LACERDA et al., 2011; LOCKER, GRUSHKA, 1987).

Munerato et al. (2005), ao realizar um estudo retrospectivo dos pacientes atendidos pelo setor de urgência da Faculdade de Odontologia da Universidade Federal do Rio Grande do Sul (UFRGS) durante o primeiro semestre de 2002, detectou que 57\% dos pacientes procuraram atendimento devido à sensação de dor, sendo que 44,11\% a dor era de origem endodôntica. De Paula et al. (2012) ao avaliar o perfil epidemiológico dos pacientes atendidos pelo Pronto Atendimento da Faculdade Federal de Juiz de Fora, relatou que a maioria (46,7\%) dos pacientes buscaram atendimento por motivo de dor, sendo que o procedimento mais comum foram restaurações definitivas (24,9\%). Martins et al. (2014) relatou a presença de dor em 80,3\% dos pacientes atendidos no serviço de urgência da FOP/UPE. Lacerda (2015) menciona que em $76 \%$ dos casos, o paciente buscou atendimento apresentando dor como queixa principal, seguida de próteses desadaptadas $(17,2 \%)$ e traumatismo dentário (6\%).

Segundo Soares e Goldberg (2011), as pulpites agudas reversíveis e irreversíveis, a pericementite apical aguda e o abscesso periapical agudo são responsáveis pela grande 
maioria dos atendimentos de urgência e exigem a intervenção endodôntica imediata. Apesar do diagnóstico mais comum envolver cárie dentária (SHQAIR et al., 2012), há uma grande incidência de traumatismos dentários, principalmente em crianças.

Paschoal et al. (2010) detectou que as lesões traumáticas em crianças de 0 a 12 anos foram responsáveis por 18,38\% dos atendimentos no setor de urgência da Faculdade de Odontologia de Bauru no período de 2004 a 2008. Sakai et al. (2005) relatam que as lesões de trauma foram responsáveis por $17,06 \%$ dos atendimentos em crianças de 0 a 15 anos. Em média, um terço da população já sofreu algum tipo de traumatismo dentário (MOULE, COHENCA, 2016). É de extrema importância o cirurgião-dentista saber como atuar em casos de traumatismo dentário, uma vez que devem ser avaliadas cautelosamente visto que o tratamento imediato tem a maior influência no prognóstico (MOULE, COHENCA, 2016).

A urgência odontológica faz parte da atenção básica à saúde, fazendo parte da rotina profissional do cirurgião-dentista, logo as universidades, como instituições de ensino e formação profissional, deve preparar e qualificar seus alunos para realizar diagnóstico preciso, bem como efetuar tais atendimentos. $\mathrm{O}$ atendimento de urgência busca, principalmente, $\mathrm{O}$ alívio da dor, eliminando imediatamente sua causa, e restauração da função dentária e encaminhamento do paciente para realização do tratamento odontológico definitivo adequado.

\section{DESENVOLVIMENTO}

Este relato de experiência descreve as atividades realizadas pelo projeto de extensão "Capacitação em Atendimento Odontológico de Urgência e Emergência” do curso de Odontologia da Universidade Federal de Santa Maria (UFSM). O projeto iniciou em março de 2017 com objetivo de proporcionar ao aluno do Curso de Odontologia maior experiência quanto a este tipo de abordagem, gerar dados para produção de conhecimento científico além de oferecer à comunidade uma opção de atendimento de urgência e emergência devido à grande demanda de um serviço especializado nesse tipo de atendimento.

Os estudantes participantes do projeto foram selecionados mediante entrevista e análise curricular, sendo que só poderiam participar acadêmicos a partir do $7^{\circ}$ semestre do. curso devido aos procedimentos realizados na clínica envolverem também a intervenção endodôntica em dentes multirradiculares. Além dos atendimentos em clínica, os alunos receberam aulas expositivas sobre assuntos relacionados à urgência e emergência em Odontologia. 
Os objetivos do projeto são realizar o tratamento de emergências e urgências odontológicas de pacientes que procuram atendimento no Curso de Odontologia da UFSM, proporcionar alívio da dor de origem endodôntica nas mais diversas situações, encaminhar os pacientes para posterior tratamento definitivo na esfera de atendimento do Curso de Odontologia, melhorar a qualidade de vida dos pacientes em função da agilidade do atendimento e oferta de maior número de vagas no que concerne neste tipo de atendimento, proporcionar ao aluno do Curso maior experiência e a realização de pesquisas na área, gerando conhecimento científico e a interação entre alunos de graduação e pós-graduação.

O projeto ocorre em dois turnos semanais nas dependências do Curso de Odontologia da UFSM. Os atendimentos são realizados por alunos de graduação sob supervisão de uma odontóloga e dois professores, para pacientes que procurarem pelo serviço de urgência e emergência do Curso, compreendendo exame clínico, anamnese, exames complementares para estabelecimento do diagnóstico e adequada intervenção.

A maioria dos tratamentos envolve intervenção endodôntica, porém também são realizados procedimentos periodontais, restauradores e cirúrgicos. O paciente, após receber devidos cuidados, é encaminhado para realização do tratamento odontológico definitivo adequado em outras Clínicas da Universidade, possibilitando promoção de saúde.

Até dezembro de 2017, com cerca de 10 meses de projeto, aproximadamente 80 pacientes receberam atendimento, obtendo suas queixas sanadas e sendo acolhidos pela Universidade. É importante ressaltar que esses pacientes, sem esse projeto, possivelmente ficariam sem assistência na Universidade, visto que o número de atendimentos de urgências nas clínicas usuais de graduação é limitado. Cerca de $77 \%$ dos atendimentos envolveram alguma intervenção endodôntica.

Desse modo, o projeto de extensão associa o ensino do estudante de graduação, a coleta de dados para pesquisa e desenvolvimento tecnológico e a interação com os membros da sociedade através dos atendimentos. "A Extensão Universitária, sob o princípio constitucional da indissociabilidade entre ensino, pesquisa e extensão, é um processo interdisciplinar, educativo, cultural, científico e político que promove a interação transformadora entre Universidade e outros setores da sociedade." (POLÍTICA NACIONAL DE EXTENSÃO, 2012, p. 42). 


\section{CONCLUSÃO}

$\mathrm{Na}$ esfera do ensino e pesquisa, o projeto de extensão proporcionou aprendizado extra aos alunos, contribuindo nas suas formações como profissionais de saúde, além da preparação para atendimentos de urgência e emergência em Odontologia. As aulas teórico-expositivas propiciaram um maior embasamento científico bem como a experiência com a montagem e análise de banco de dados em saúde.

O caráter extensionista da ação tem sua base no aumento da disponibilidade do atendimento de urgências e emergência à população visto que, conseguir assistência na Universidade antes da existência do projeto, era altamente dificultoso fazendo com que esses pacientes ficassem desamparados.

Através da ação de extensão foi possível estabelecer um vínculo com a comunidade e a universidade, facilitando o acesso da população ao serviço e melhorando sua qualidade de vida e desenvolvendo um conhecimento prático, teórico e científico para os alunos do Curso de Odontologia da UFSM. 


\section{REFERÊNCIAS}

ALBUQUERQUE, Y.E. et al. Perfil do atendimento odontológico no Serviço de Urgência para crianças e adolescentes da Faculdade de Odontologia de Araraquara (FOAr) - UNESP. Revista de Odontologia da UNESP, v. 45, n.2, p. 115-120, 2016.

CAVALHEIRO, C.H. et al. Dental pain, use of dental services and oral health-related quality of life in southern Brazil. Braz. Oral Res., v. 30, n. 1, 2015.

CONSTANTE, H.M. et al. Socio-demographic and behavioural inequalities in the impact of dental pain among adults: a population-based study. Community Dentistry and Oral Epidemiology, v. 40, n.6, p. 498-506, 2012.

DA SILVA, J.L. Atendimentos de Urgência nas Faculdades de Odontologia do Brasil. Trabalho de Conclusão de Curso de Especialização (Especialização em Atenção Especializada em Saúde) - Universidade Federal do Rio Grande do Sul, Porto Alegre. 2015.

DE PAULA, JS. et al. Perfil epidemiológico dos pacientes atendidos no Pronto Atendimento da Faculdade de Odontologia da Universidade Federal de Juiz de Fora. Arq Odontol., v. 48, n. 4, p. 257-262, 2012.

FORPROEX. Política Nacional De Extensão Universitária. 2012. Disponível em: http://proex.ufsc.br/files/2016/04/Pol\%C3\%ADtica-Nacional-de-Extens\%C3\%A3o-Universit \%C3\%A1ria-e-book.pdf. Acesso em 5 abril 2018.

LACERDA, J.T. et al. Prevalência da dor orofacial e seu impacto no desempenho diário em trabalhadores das indústrias têxteis do município de Laguna, SC. Ciência \& Saúde Coletiva, v. 16, n. 1, p. 4275-4282, 2011.

LOCKER, D., GRUSHKA, M. The Impact of Dental and Facial Pain. J Dent. Res., v. 66, n. 9, p. 1414-1417, 1987.

MARTINS, E.P. et al. Estudo epidemiológico de urgências odontológicas da FOP/UPE. RFO, v. 19, n. 3, p. 316-322, 2014.

MOULE, A. COHENCA, N. Emergency assessment and treatment planning for traumatic dental injuries. Australian Dental Journal, v. 61, n. 1, p. 21-38, 2016.

MUNERATO, M.C., FIAMINGHI, D.L., PETRY, P.C. Urgências em Odontologia: um estudo retrospectivo. R. Fac. Odonto., v. 46, n. 1, p. 90-95, 2005.

PASCHOAL, M.A.B. et al. Perfil de tratamento de urgência de crianças de 0 a 12 anos de idade, atendidas no serviço de Urgência da Faculdade de Odontologia de Bauru da Universidade de São Paulo. Odontol Clin Cient, v. 9, n. 3, 2010.

SAKAI, V.T. et al. Urgency Treatment Profile of 0 to 15 year-old children assisted at Urgency Dental Service from Bauru Dental School, University Of São Paulo. Journal of Applied

Oral Science, v. 13, n. 4, 2005. 
SOARES, I.J., GOLDBERG, F. Endodontia: Técnicas e fundamentos. 2.ed. Porto Alegre. Artmed, 2011.

SHQAIR, A.Q. et al. Dental emergencies in a university pediatric dentistry clinic: a retrospective study. Braz Oral Res., v. 26, n. 1, 2012. 